\title{
A New Method for Prediction of Water Flooding Development Index
}

\author{
Jinshan Liu \\ Geological Team, No.3 Oil Recovery Plant, Daqing Oil Field Corp. Ltd., Daqing 163113, China \\ Liujinshan2008@163.com
}

Keywords: Exponential Decline Model, Type C Water Flooding Feature Curve, Water Flooding Development Index, Prediction.

\begin{abstract}
This paper which combined exponential decline model with Type $\mathrm{C}$ water flooding feature curve, presented a simultaneous solution for index prediction of water flooding development, and the method overcame their shortcomings which curves cannot predict the relationship of development index and times in the dynamic prediction. While water cut, liquid production capacity, water production capacity, cumulative water production and cumulative liquid production of oilfield were unable to predict by exponential decline model. Meanwhile, the method of resultant is adopted to predict the water drive development indexes of the layer series of development among the basic well pattern at Sabei development area. Results suggest that the new method is practical and effective.
\end{abstract}

\section{Introduction}

Type C water flooding feature curve was an important prediction method in petroleum reservoir engineering, which was used to predict relationship of cumulative water production and cumulative oil production, cumulative water-oil ratio and cumulative liquid production. Though exponential decline model was adopted to forecast variable characteristics that oil production varied with development time, it cannot get with the prediction of water cut, water production capacity, liquid production capacity, cumulative water production and cumulative liquid production. While the prediction of these development index were just what water flooding oil field need. This paper which combined exponential decline model with Type $\mathrm{C}$ water flooding feature curve, presented a simultaneous synchronous solution for index prediction of water flooding development. The method was used to overcome limitations of the two predictions, and keep their original function. The method of resultant was adopted to predict the water drive development indexes of the layer series of development among the basic well pattern at Sabei development area, and got a statistically significant prediction rate.

\section{Guiding methods}

Type C water flooding feature curve was proposed by Nazarov, Sipparchev and so on. The formula was deduced theoretically by literature [1]

$$
\frac{L_{p}}{N_{p}}=a+b L_{p}
$$

$L_{p^{--}}$cumulative liquid production, $10^{4} \mathrm{t}$

$N_{p^{--}}$cumulative oil production, $10^{4} \mathrm{t}$

The formula of water cut and cumulative oil production were predicted by Type $\mathrm{C}$ water flooding feature curve

$$
f_{w}=1-\frac{\left(1-b N_{p}\right)^{2}}{a}
$$

$a$--straight intercept of type $\mathrm{C}$ water flooding feature curve

$b$--straight slope of type $\mathrm{C}$ water flooding feature curve

Exponential Decline Prediction Model [2-4], had predicted function of oil production, cumulative oil production and recoverable reserve. And the formula of oil production and time by model declined 
$Q_{t}=Q_{i} e^{-D_{i} t}$

$Q_{i^{--o i l ~}}$ production of preliminary decline, $10^{4} \mathrm{t} / \mathrm{a}$

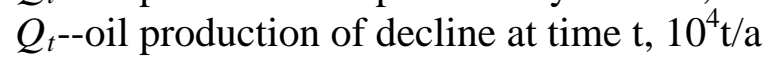

$D_{i}$--initial decline rate

Log and simplify both sides

$D_{i}=\frac{\lg Q_{i}-\lg Q_{t}}{t \lg e}$

The formula of cumulative oil production varied with time

$N_{p t}=N_{p i}+\frac{Q_{i}}{D_{i}}\left[1-\exp \left(-D_{i} t\right)\right]$

$N_{p i^{--}}$cumulative oil production of preliminary decline, $10^{4} \mathrm{t}$

$N_{p t^{--}}$cumulative oil production of decline at time t, $10^{4} \mathrm{t}$

As $\mathrm{t} \rightarrow \infty$, recoverable reserve can be obtained

$N_{R}=N_{p i}+\frac{Q_{i}}{D_{i}}$

Plug (5) into (2), the integrated method of water cut prediction can be obtained

$f_{w}=1-\frac{\left\{1-\frac{b\left[N_{p i}+Q_{i}\left(1-\exp \left(-D_{i} t\right)\right)\right]}{D_{i}}\right\}^{2}}{a}$

From (3), (5) and (7), the prediction of oil production, cumulative oil production and cumulative water production can be obtained

$$
\begin{aligned}
Q_{w} & =Q_{o}\left(\frac{f_{w}}{1-f_{w}}\right) \\
Q_{L} & =Q_{o}\left(\frac{1}{1-f_{w}}\right) \\
W_{p} & =\sum_{0}^{t} Q_{w} \\
L_{p} & =\sum_{0}^{t} Q_{L}
\end{aligned}
$$

\section{Parameter solution}

According to practical oil production information, annual oil production varied with time would be drawn on semilog coordinate at first, ascertaining oil production of preliminary decline, and decline rate $D_{i}$ can be obtained from formula(4).

Because of prediction of oilfield development by means of exponential decline prediction model was after initial decreasing point, the prediction by simultaneous solution was as well. In order to make type $C$ water flooding feature curve predict accurately and reliably, it is better to neglect $L_{p}$ and $N_{p}$ in calculating parameter $a$ and $b$, while put $L_{p} / \mathrm{N}_{p}$ and $L_{p}$ in (2) according to linear regression, so parameter $\mathrm{a}$ and $\mathrm{b}$ of the feature curve were determined.

By putting $D_{i}, a$ and $b$ into (3),(5),(6),(7),(8),(9),(10) and (11),obtained oilfield recoverable reserves and oil production, water production, liquid production, cumulative oil production, cumulative water production, cumulative liquid production, water cut for each phase of oilfield development.

\section{Application example}

The Beisandong basic well pattern at Sabei development area went into operation in 1973, the logarithm of oil production of water flooding each year were drawn on the Fig.1 regarding time as x-coordinate. It is shown that, production decline rate changed linearly at the beginning of 1994, and oil production of water flooding was declined in exponent regularity. If $Q_{i}=97.2628 \times 10^{4} \mathrm{t}, \mathrm{t}=19 \mathrm{a}$, 
corresponded to $Q_{t}=17.09 \times 10^{4} \mathrm{t}, N_{p i}=1971.62 \times 10^{4} \mathrm{t}, N_{p t}=2762.23 \times 10^{4} \mathrm{t}$, put above data into (4), $D_{i}=0.0915 \mathrm{a}^{-1}$ was obtained. And put $L_{p} / \mathrm{N}_{p}$ after 1986, corresponding $L_{p}$ in (2) according to linear regression, $a=0.9575, b=2.9102 \times 10^{-4}$ were determined. Plug $D_{i}$ and $N_{p i}$ into (6)

$$
N_{R}=1971.62+\frac{97.2628}{0.09151}=3034.49 \times 10^{4}(\mathrm{t})
$$

Plug $D_{i}, a$ and $b$ into (3), (5) and (7) respectively, the relationship of theoretical annual oil production after water-flooding production decline, cumulative oil production and water cut would be obtained, and the relationship was used to predict Beisandong basic well pattern development layer series from 1994

$$
\begin{aligned}
& Q_{o}=Q_{t}=97.2628 e^{-0.09151 t} \\
& N_{p}=\frac{97.2628}{0.09151}[1-\exp (-0.09151 t)]+1971.62 \\
& f_{w}=1-\frac{\left\{1-\frac{\left.2.9102 \times 10^{-4}\left[1971.62+97.2628\left(1-\exp \left(-0.0915_{i} t\right)\right)\right]\right\}^{2}}{0.0915}\right.}{0.9575}
\end{aligned}
$$

Given different development time $t$, theoretical annual oil production after water flooding production decline, cumulative oil production and water cut were predicted by (12), (13) and (14). In drawing fig.2, fig.3, fig.4, there was curves as prediction curves and points as actual values, it is shown that the prediction agreed well with actual values.

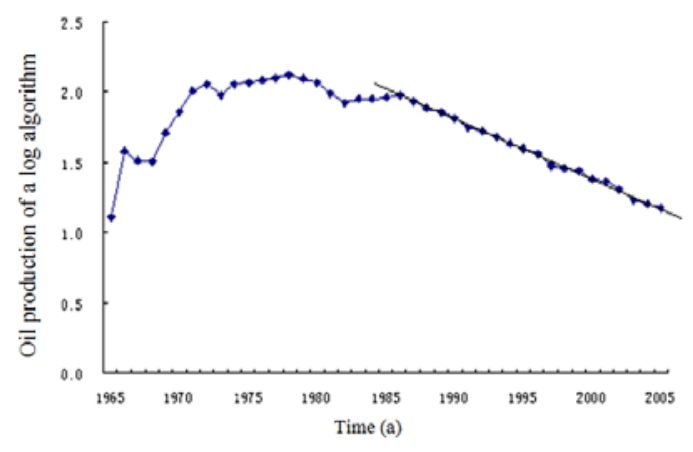

Fig.1 Semi-logarithmic of annual oil production

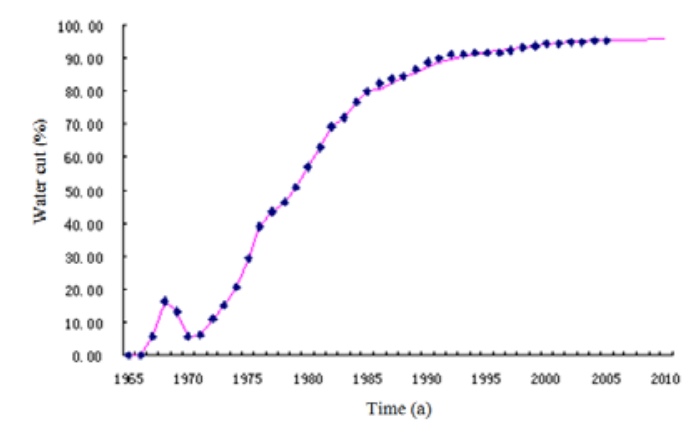

Fig.3 Contrast with forecast curves and actual dat of water cut

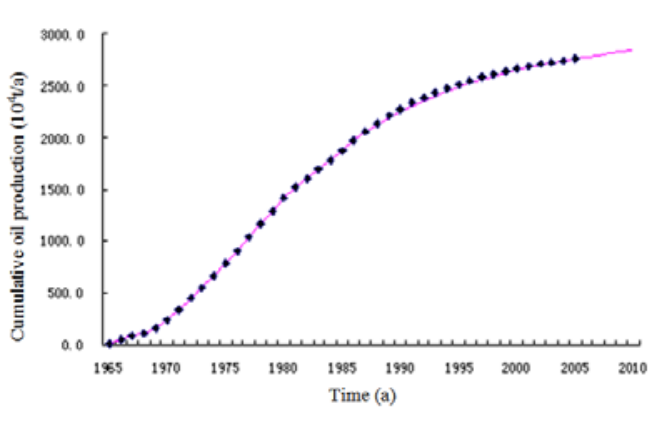

Fig.2 Contrast with forecast curves and actual data of cumulative oil production

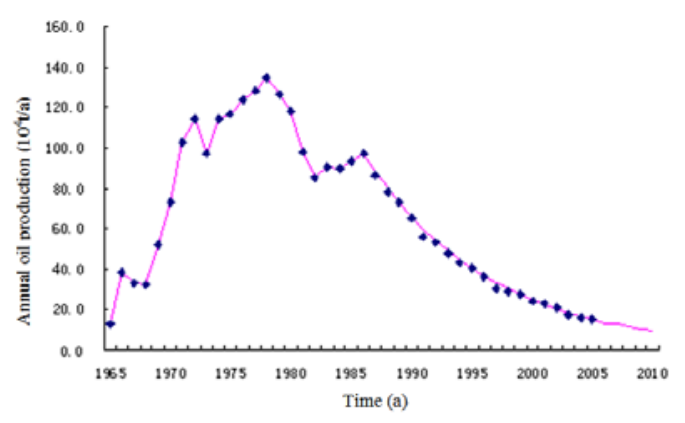

Fig.4 Contrast with forecast curves and actual data of annual oil production

\section{Conclusion}

(1)Basing on the analytical processing of water drive oil production among the basic well pattern at Beisandong development area, this paper determined that the law of diminishing water flooding almost conformed with exponential decline.

(2) The simultaneous solution of exponential decline model with Type $\mathrm{C}$ water flooding feature curve presented in this article, was able to forecast development index in different period of decline 
stage at the late development stage of an oilfield, overcame limitations of two models when they used alone.

(3)The method of resultant was adopted to predict the water drive development indexes of the layer series of development among the basic well pattern at Sabei development area, and it was shown that a high degree of fitness in case of predictions and actral values of development index at decline stage.

\section{References}

[1]Y.Q. Chen. Petroleum Exploration and Development, Vol. 22 (1995) No.3, p. 63-68.

[2]T.P. Chen, Jingbang Hu. Petroleum Engineering. (BeiJing Petroleum Industry Press, 1999).p. 461-465.

[3]Zhen Wang. Natural Gas Industry, Vol. 23 (2003) No.6, p. 62-64.

[4]Q.T. Yu. XinJiang Petroleum Geology, Vol. 15 (1994) No.1, p. 49-55. 